\title{
QUALITY IMPROVEMENT IN AN INVENTORY MODEL WITH FINITE-RANGE STOCHASTIC LEAD TIMES
}

\author{
JAVAD PAKNEJAD, FARROKH NASRI, AND JOHN F. AFFISCO
}

Received 10 January 2002 and in revised form 1 May 2002

Typically, traditional inventory models operate under the assumption of perfect quality. In this paper we modify an inventory model with finite-range stochastic lead time to allow for a random number of defective units in a lot. However, there is an extra cost for holding the defective items in the lot for the period before it is returned to the supplier. This paper also considers the option of investment to improve quality. Closed-form relationships are obtained for a quality-adjusted model as well as a quality improvement model. Numerical examples confirm that the option of investment in quality improvement results in significant cost savings. Sensitivity analysis shows that the quality improvement model is robust.

\section{Introduction}

Lot size research owes its beginnings to the traditional square root EOQ formula. This relationship is the result of classical optimization of inventory related costs under a series of highly restrictive assumptions. Among which are instantaneous replenishment, constant deterministic demand and lead-time, and perfect quality of inventory items. More realistic cases ensue when these assumptions are relaxed. One such case that has appeared in the literature is that which allows for variability in either demand or lead-time, or both.

Gross and Soriano [2] and Vinson [15], among others, demonstrate that lead-time variation has a major impact on lot size and inventory costs. Furthermore, they indicate that an inventory system is more sensitive to lead time variation than to demand variation. The problem of the EOQ model with stochastic lead-time has been considered by several additional authors including Liberatore [5], Sphicas [13], and Sphicas and Nasri [14]. In this last work the authors derive a closed-form expression for EOQ with backorders when the range of the lead-time distribution has a finite upper bound. In this formulation all units are assumed to be of perfect quality.

Concurrently work has appeared in the literature that relaxes the perfect quality assumption. Rosenblatt and Lee [12] have investigated the effect of process quality on lot 
size in the classical economic manufacturing quantity model (EMQ). Porteus [11] introduced a modified EMQ model that indicates a significant relationship between quality and lot size. In both Rosenblatt and Lee [12] and Porteus [10] the optimal lot size is shown to be smaller than that of the EMQ model. In these works, the deterioration of the production system is assumed to follow a random process.

Cheng [1] develops a model that integrates quality considerations with EPQ. The author assumes that unit production cost increases with increases in process capability and quality assurance expenses. Classical optimization results in closed forms for the optimal lot size and optimal expected fraction acceptable. The optimal lot size is intuitively appealing since it indicates an inverse relationship between lot size and process capability.

While this previous work relaxes the perfect quality assumption, it also considers demand to be deterministic. A number of authors have investigated the impact of quality on lot size under conditions of stochastic demand and or stochastic lead-time. Moinzadeh and Lee [6] have studied the effect of defective items on the operating characteristics of a discontinuous review inventory system with Poisson demand and constant lead-time. Paknejad et al. [7] present a quality-adjusted lot-sizing model with stochastic demand and constant lead-time. Specifically, they investigate the case of continuous review $(s, Q)$ models in which an order of size $Q$ is placed each time the inventory position (based on nondefective items) reaches the order point $s$. Results indicate that as the probability of defective items increases, for a given constant lead-time, the optimal lot size and the optimal reorder point both increase significantly. Further, for a given defective probability, the lot size and the reorder point increase substantially as the lead-time increases. This indicates that improving quality when lead-time is constant should result in smaller lot sizes.

Variations in lead-time can occur for purchased items and for those that are manufactured in-house. A major factor related to these variations is quality problems. Heard and Plossl [4] portray high lead-time variability as a major reason for a plant's inability to achieve inventory goals, and to incur longer average throughput. This suggests that it would be worthwhile to investigate the relationship between quality and lead-time variability, and their impact on lot size and inventory cost. The purpose of this paper is to begin the analytical investigation of these relationships.

\section{The basic model}

The basic model considered in this paper is the classic EOQ with constant noninterchangeable demand, backorders, and finite-range stochastic lead-time, developed by Sphicas and Nasri [14]. The cost function of this model is

$$
\begin{aligned}
\operatorname{AC}(q, t)= & K / q+\int_{\alpha}^{t}\{h D(t-r)+h D q / 2\} g(r) d r \\
& +1 / q \int_{t}^{t+q}\left\{p D(r-t)^{2} / 2+h D(t+q-r)^{2} / 2\right\} g(r) d r \\
& +\int_{t+q}^{\beta}\{p D q / 2+p D(r-t-q)\} g(r) d r
\end{aligned}
$$


where

$D=$ demand per unit time (in units),

$K=$ setup cost per setup,

$h=$ holding cost per (nondefective) unit per unit time,

$p=$ backorder cost per unit per unit time,

$\mathrm{AC}(q, t)=$ expected average cost per unit time,

$Q=$ lot size per order,

$q=Q / D=$ number of time units of demand satisfied by each order,

$t=$ time differential between placing an order and the start of $q$ time units that

will be satisfied by a given order,

$r=$ lead time in units of time (a random variable),

$g(r)=$ lead-time probability density function,

$\alpha=$ lower bound of lead-time distribution,

$\beta=$ upper bound of lead-time distribution,

$\mu=$ mean of lead-time distribution.

They show that when $t \leq \alpha$ and $t+q \geq \beta$, orders may not cross each other and the above cost function reduces to

$$
\mathrm{AC}(q, t)=\frac{K}{q}+\frac{1}{q} \int_{\alpha}^{\beta}\left\{p D(r-t)^{2} / 2+h D(t+q-r)^{2} / 2\right\} g(r) d r .
$$

In this case, the optimal values of the decision variables, $q *$ and $t^{*}$, and the resulting optimal lot size, $Q^{*}$, and the optimal average cost per unit time, $\operatorname{AC}^{*}(q, t)$, are given by

$$
\begin{gathered}
q^{*}=\sqrt{\left[\frac{2 K}{D}+(h+p) V\right]\left(\frac{1}{h}+\frac{1}{p}\right)}, \\
t^{*}=\mu-\sqrt{\Omega\left[\frac{2 K}{(h+p) D}+V\right]}, \\
Q^{*}=\sqrt{\left[2 D K+V D^{2}(h+p)\right]\left(\frac{1}{h}+\frac{1}{p}\right)}, \\
\operatorname{AC}^{*}(q, t)=\sqrt{\left[2 D K+V D^{2}(h+p)\right] /\left(\frac{1}{h}+\frac{1}{p}\right)},
\end{gathered}
$$

where $\Omega=h / p$ and $V$ is the variance of the lead-time. Note that (2.5) is the stochastic generalization of EOQ when shortages are allowed. Sphicas and Nasri [14] proved that in terms of the parameters of the model, crossover may not occur if and only if $k \geq k_{2}$, where

$$
\begin{gathered}
k=2 K /(h+p) D, \\
k_{2}=(\mu-\alpha)^{2} / \Omega-V, \quad \text { if } \Omega \leq(\mu-\alpha) /(B-\mu), \\
k_{2}=\Omega(\mu-\beta)^{2}-V, \quad \text { if } \Omega \geq(\mu-\alpha) /(B-\mu) .
\end{gathered}
$$

This formulation assumes that all the units produced by the vendor, in response to purchaser's order, are nondefective. In the next section, we relax this assumption and extend 
the stochastic generalization of the EOQ model with no cross-over of orders by allowing the possibility that each lot may contain a random number of defective units. Finally, we investigate the effect of investing to improve the quality on the optimal values of the decision variables in the quality-adjusted model.

\section{Quality-adjusted model and assumptions}

In this section we modify the basic model with no cross-over of orders as follows. First, we assume that each lot contains a random number of defective units. Upon arrival, the purchaser inspects the entire lot piece by piece. The purchaser removes the defective units from the lot and returns them to the vendor at the time of next delivery. It is assumed that the vendor picks up the inspection cost incurred by the purchaser. The purchaser's inventory system, however, incurs an extra cost for holding the defective units in stock until the time they are returned to the vendor. We use the following additional notations in our presentation,

$Y=$ number of nondefective units in a lot, a random variable,

$z=Y / D=$ number of time units of demand satisfied by nondefective units in each lot (cycle time), a random variable,

$h=$ nondefective holding cost per unit per unit time,

$h^{\prime}=$ defective holding cost per unit per unit time,

$C(z)=$ cost per cycle,

$E(C \mid Q)=$ expected value of $C(z)$ given that a lot of size $Q$ is ordered,

$\mathrm{EA}(C \mid Q)=$ expected average cost per unit time given that a lot of size $Q$ is ordered,

$E(z \mid Q)=$ expected cycle time, first moment of $z$, given that a lot of size $Q$ is ordered,

$E\left(z^{2} \mid Q\right)=$ second moment of $z$ given that a lot of size $Q$ is ordered.

The cost per cycle is

$$
C(z)=K+z \int_{\alpha}^{\beta}\left\{p D(r-t)^{2} / 2 z+h D(t+z-r)^{2} / 2 z\right\} g(r) d r+h^{\prime} D z(q-z) .
$$

The expected cycle cost is

$$
\begin{aligned}
E(C \mid Q)= & K+\frac{D}{2}\left\{(h+p)\left[V+(t-\mu)^{2}\right]+2 h(t-\mu) E(z \mid Q)+h E\left(z^{2} \mid Q\right)\right\} \\
& +h^{\prime} D E(z \mid Q)[q-E(z \mid Q)] .
\end{aligned}
$$

Multiplying (3.2) by the expected number of cycles per unit time, $1 / E(z \mid Q)$, we have the expected average cost per unit time as follows:

$$
\begin{aligned}
\mathrm{EA}(C \mid Q)= & \frac{K}{E(z \mid Q)}+\frac{D}{2 E(z \mid Q)}(h+p)\left[V+(t-\mu)^{2}\right]+D h(t-\mu) \\
& +\frac{D h}{2} \frac{E\left(z^{2} \mid Q\right)}{E(z \mid Q)}+h^{\prime} D q-h^{\prime} D E(z \mid Q)
\end{aligned}
$$


Assuming that the number of nondefective units in a lot of size $Q$ can be described by a binomial random variable with parameters $Q$ and $(1-\theta)$, then

$$
\begin{gathered}
E(z \mid Q)=q /(1+\rho), \\
E\left(z^{2} \mid Q\right)=q(\rho+D q) /(1+\rho)^{2} D,
\end{gathered}
$$

where $\rho$ is the ratio of the probability of a defective unit to the probability of a nondefective unit. That is,

$$
\rho=\frac{\theta}{1-\theta}
$$

Substituting (3.4) into (3.3), we have

$$
\begin{aligned}
\mathrm{EA}(C \mid Q)= & \frac{K(1+\rho)}{q}+\frac{D(1+\rho)}{2 q}(h+p)\left[V+(t-\mu)^{2}\right]+D h(t-\mu) \\
& +\frac{D h}{2}\left[\frac{\rho+D q}{(1+\rho) D}\right]+h^{\prime} D q-\frac{h^{\prime} D q}{1+\rho} .
\end{aligned}
$$

The optimal values for the decision variables, $q_{\text {adj }}^{*}, t_{\text {adj }}^{*}$ and the resulting optimal lot size, $Q_{\text {adj }}^{*}$, and optimal expected average cost per unit time, $\mathrm{EA}_{\mathrm{adj}}^{*}(C \mid Q)$, are found using calculus as follows:

$$
\begin{aligned}
q_{\text {adj }}^{*} & =\frac{1+\rho}{\eta} q^{*}, \\
t_{\text {adj }}^{*} & =\mu+\frac{t^{*}-\mu}{\eta}, \\
Q_{\text {adj }}^{*} & =\frac{1+\rho}{\eta} Q^{*}, \\
E_{\text {adj }}^{*}(C \mid Q) & =\frac{h}{2}\left(\frac{\rho}{1+\rho}\right)+\eta \operatorname{AC}^{*}(q, t),
\end{aligned}
$$

where

$$
\eta=\left[1+2 h^{\prime} \rho\left(\frac{1}{h}+\frac{1}{p}\right)\right]^{1 / 2},
$$

and $q^{*}, t^{*}, Q^{*}$, and $\operatorname{AC}^{*}(q, t)$ are given in (2.3), (2.4), (2.5), and (2.6), respectively. Note that in (3.7) through (3.10), if the quality parameter $\rho=0$, then quality is perfect and the quality-adjusted model with finite-range stochastic lead-time simply reduces to Sphicas and Nasri's basic model with no cross-over of orders expressed in (2.3) through (2.6). In (3.9) it would be of interest to know under what conditions $Q_{\text {adj }}^{*}$ will be larger, smaller, or equal to $Q^{*}$. To answer this question we form the ratio of $Q_{\mathrm{adj}}^{*} / Q^{*}$ as follows:

$$
f(\rho)=\frac{Q_{\text {adj }}^{*}}{Q^{*}}=\frac{1+\rho}{\left[1+2 h^{\prime} \rho(1 / h+1 / p)\right]^{1 / 2}} .
$$


Using methods of calculus it can easily be shown that the function presented in (3.12) is convex in $\rho$. Further investigation of this function reveals that it reaches its minimum at

$$
\rho_{\min }=1-\frac{1}{h^{\prime}(1 / h+1 / p)}
$$

Inspecting this minimum indicates that there are two distinct scenarios for the relationship between $Q_{\mathrm{adj}}^{*}$ and $Q^{*}$. First, when $h^{\prime}(1 / h+1 / p) \leq 1$ and $\rho>0$ then $Q_{\text {adj }}^{*}>Q^{*}$. Second, when $h^{\prime}(1 / h+1 / p)>1$ three possibilities emerge:

(i) if $0<\rho<2\left[h^{\prime}(1 / h+1 / p)-1\right]$ then $Q_{\text {adj }}^{*}<Q^{*}$,

(ii) if $\rho=2\left[h^{\prime}(1 / h+1 / p)-1\right]$ then $Q_{\text {adj }}^{*}=Q^{*}$,

(iii) if $\rho>2\left[h^{\prime}(1 / h+1 / p)-1\right]$ then $Q_{\text {adj }}^{*}>Q^{*}$.

\section{The approximately optimal quality model}

The policy variables in (3.6) are $q$ and $t$ for a fixed quality parameter value, $\rho$. In this paper, as in Porteus [11] and Paknejad et al. [8], we assume that the option of investing to reduce $\rho$ is available. Thus we consider $\rho$ to be a decision variable and aim at minimizing the expected average cost per unit time composed of investment to change $\rho$, ordering, shortage, nondefective holding, and defective holding costs. Specifically, we seek to minimize

$$
\operatorname{EAC}(q, t, \rho)=i a_{\rho}(\rho)+\operatorname{EA}(C \mid Q)
$$

subject to

$$
0<\rho \leq \rho_{0},
$$

where $i$ is the cost of capital, $a_{\rho}(\rho)$ is a convex and strictly decreasing function of $\rho$ representing the investment cost needed to change the quality parameter to the level $\rho$, $\mathrm{EA}(C \mid Q)$ is the sum of inventory related costs given in (3.6), and $\rho_{0}$ is the original quality parameter.

One reasonable way of dealing with this optimization problem is to use a rather nonconventional approach suggested by Porteus [9]. In this case, we fix $\rho$, optimize over $q$ and $t$ to obtain $q_{\text {adj }}^{*}(\rho)$ and $t_{\text {adj }}^{*}(\rho)$, and then optimize over $\rho$. That is, we seek to minimize

$$
w(\rho)=i a_{\rho}(\rho)+C_{\rho}(\rho)
$$

where

$$
C_{\rho}(\rho)=\mathrm{EA}_{\mathrm{adj}}^{*}(C, Q) \simeq \eta \mathrm{AC}^{*}(q, t),
$$

where $\mathrm{EA}_{\text {adj }}^{*}(C, Q)$ is given by $(3.10), \eta$ by $(3.11)$, and $\mathrm{AC}^{*}(q, t)$ by $(2.6)$. Note that $C_{\rho}(\rho)$ is assumed to be approximately equal to the second component of (3.10). We have ignored the first component of (3.10) which is less than one-half of cost of holding only one nondefective unit in inventory per unit time. 
Of course if the optimal $\rho$ obtained in this way does not satisfy restriction (4.2), we should not make any investment and the results of the quality-adjusted model of the previous section hold. It should be pointed out that it may not always be possible to carry out the minimization except for some special cases of $a_{\rho}(\rho)$. The following section treats one of these cases.

4.1. The logarithmic investment function case. This particular function is used in previous research by Porteus $[9,10]$ and Paknejad et al. $[7,8]$ dealing with quality improvement as well as setup cost reduction. It is consistent with the Japanese experience as reported in Hall [3]. In this case the following form of investment function is used

$$
a_{\rho}(\rho)=\frac{1}{\delta} \operatorname{Ln} \frac{\rho_{0}}{\rho} \quad \text { for } 0<\rho \leq \rho_{0}
$$

where $\delta$ is the percentage decrease in $\rho$ per dollar increase in $a_{\rho}$. Here our main objective is to minimize the following, which is the result of substituting (4.5) into (4.3)

$$
w(\rho)=\frac{i}{\delta} \operatorname{Ln} \frac{\rho_{0}}{\rho}+\eta \mathrm{AC}^{*}(q, t)
$$

THEOREM 4.1. If $\delta$ is strictly positive, then the following hold.

(a) The optimal quality parameter,

$$
\rho_{\mathrm{imp}}^{*}=\min \left\{\rho_{0}, \rho_{\mathrm{imp}}\right\}
$$

where

$$
\rho_{\mathrm{imp}}=\frac{1}{h^{\prime}}\left(\frac{1}{h}+\frac{1}{p}\right)\left(\frac{i}{\delta Q^{*}}\right)^{2}\left[1+\sqrt{1+\left(\frac{\delta Q^{*}}{i(1 / h+1 / p)}\right)^{2}}\right]
$$

and $Q^{*}$ is given by (2.5).

(b) The resulting approximately optimal number of time units of demand satisfied by each order, $q_{\mathrm{imp}}^{*}$, and the approximately optimal time differential between placing an order and the start of $q_{\mathrm{imp}}^{*}$ time units that will be satisfied by a given order, $t_{\mathrm{imp}}^{*}$, are given by

$$
\begin{aligned}
q_{\mathrm{imp}}^{*} & =\frac{1+\rho_{\mathrm{imp}}^{*}}{\left[1+2 h^{\prime} \rho_{\mathrm{imp}}^{*}(1 / h+1 / p)\right]^{1 / 2}} q^{*}, \\
t_{\mathrm{imp}}^{*} & =\mu+\frac{\left(t^{*}-\mu\right)}{\left[1+2 h^{\prime} \rho_{\mathrm{imp}}^{*}(1 / h+1 / p)\right]^{1 / 2}} .
\end{aligned}
$$


(c) The resulting approximately optimal lot size, $Q_{i m p}^{*}$, and the approximately optimal average cost per unit time, $w_{\mathrm{imp}}^{*}$, are

$$
\begin{gathered}
Q_{\mathrm{imp}}^{*}=\frac{1+\rho_{\mathrm{imp}}^{*}}{\left[1+2 h^{\prime} \rho_{\mathrm{imp}}^{*}(1 / h+1 / p)\right]^{1 / 2}} Q^{*}, \\
w_{\mathrm{imp}}^{*}=\frac{i}{\delta} \operatorname{Ln} \frac{\rho_{0}}{\rho_{\mathrm{imp}}^{*}}+\left[1+2 h^{\prime} \rho_{\mathrm{imp}}^{*}\left(\frac{1}{h}+\frac{1}{p}\right)\right]^{1 / 2} \operatorname{AC}^{*}(q, t) .
\end{gathered}
$$

Proof. Part (a) is the direct result of minimizing (4.6) with respect to $\rho$ for the two separate cases of $0<\rho \leq \rho_{0}$ and $\rho \geq \rho_{0}$. If $0<\rho_{\text {imp }}<\infty$ minimizes the $w$ of (4.6), then it is necessary that $\rho_{\text {imp }}$ satisfy

$$
\frac{\partial w}{\partial \rho}=-\frac{i}{\delta \rho}+h^{\prime} \frac{Q^{*}}{\eta}=0 .
$$

Solving (4.11) gives (4.8). To prove that $\rho_{\text {imp }}$ is a relative minimum, it is sufficient to show that $\partial^{2} w / \partial \rho^{2}$ is strictly positive when $\rho=\rho_{\text {imp }}$, that is,

$$
\frac{\partial^{2} w}{\partial \rho^{2}}=\frac{i}{\delta \rho^{2}}-h^{\prime 2}\left(\frac{1}{h}+\frac{1}{p}\right) \frac{Q^{*}}{\eta^{3}}>0 .
$$

Finding $i / \delta \rho$ from (4.11) and substituting it into (4.12), and after some manipulations, we find that when

$$
\rho_{\text {imp }}>\frac{-1}{h^{\prime}(1 / h+1 / p)},
$$

then we have a relative minimum. Since the right-hand side of (4.13) is negative, the inequality is satisfied. Hence, part (a) holds. (b) and (c) are the result of substituting $\rho_{\mathrm{imp}}^{*}$ into appropriate expressions.

\section{Numerical examples}

Consider an example where the following parameters are known: $D=5200$ units/year, $K=\$ 500 /$ setup, $h=\$ 10 /$ unit-year, $p=\$ 20 /$ unit-year, $h^{\prime}=\$ 5 /$ unit-year, $i=0.10$, and $\delta=0.0005$. Table 5.1 presents the results of calculations for the economic order quantity under three scenarios: the EOQ with uniformly distributed lead-time (EOQ-SLT) over a one week interval, the quality-adjusted EOQ (SLT-QA) with uniformly distributed leadtime over a one week interval, and the EOQ including investment in quality improvement for the case of uniformly distributed lead time over a one week interval (SLT-QA-Imp). It is interesting to note that SLT-QA is an upper bound for total cost and economic order quantity for the problem. Further, it can be seen from (3.7), (3.8), (3.9), and (3.10) that as the proportion of defectives, $\theta$, approaches zero, the results become identical to Sphicas and Nasri [14].

Table 5.2 presents the results of additional calculations aimed at determining the impact of lead-time variability on the model developed in this paper. Specifically, the 
Table 5.1. Comparative results for uniform lead-time distribution.

\begin{tabular}{lccc}
\hline Variable & EOQ-SLT & SLT-QA & SLT-QA-Imp \\
\hline$Q$ (units) & 885.30 & 943.73 & 895.80 \\
$\theta$ & - & 0.2 & 0.045 \\
$\rho$ & - & 0.25 & 0.047 \\
TC $(\$)$ & $5,901.97$ & $6,920.67$ & $6,105.36$ \\
$\%$ TC Savings over SLT-QA & - & - & 11.78 \\
\hline
\end{tabular}

$K=500, D=5200, h=10, p=20, h^{\prime}=5, \mu=0.009615, V=0.0000308, i=0.1, \delta=0.0005$.

Table 5.2. Optimal value for various uniform lead-time variabilities.

\begin{tabular}{|c|c|c|c|c|c|c|c|c|c|c|}
\hline \multirow{3}{*}{ Variable } & \multicolumn{10}{|c|}{ Lead-time interval (weeks) } \\
\hline & \multicolumn{2}{|c|}{1} & \multicolumn{2}{|c|}{2} & \multicolumn{2}{|c|}{3} & \multicolumn{2}{|c|}{4} & \multicolumn{2}{|c|}{5} \\
\hline & QA & $\operatorname{Imp}$ & $\mathrm{QA}$ & $\operatorname{Imp}$ & QA & $\operatorname{Imp}$ & QA & $\operatorname{Imp}$ & QA & $\operatorname{Imp}$ \\
\hline$Q$ (units) & 943.73 & 895.80 & 950.48 & 902.21 & 961.62 & 912.79 & 977.01 & 927.39 & 996.44 & 945.84 \\
\hline$\theta$ & 0.2 & 0.0447 & 0.2 & 0.0443 & 0.2 & 0.0438 & 0.2 & 0.0432 & 0.2 & 0.0423 \\
\hline$\rho$ & 0.25 & 0.0467 & 0.25 & 0.0464 & 0.25 & 0.0458 & 0.25 & 0.0451 & 0.25 & 0.0442 \\
\hline $\mathrm{TC}(\$)$ & 6290.68 & 6105.36 & 6970.17 & 6147.55 & 7051.89 & 6217.20 & 7164.73 & 6313.37 & 7307.25 & 6434.84 \\
\hline $\begin{array}{l}\text { \%TC Sav- } \\
\text { ings over } \\
\text { SLT-QA }\end{array}$ & - & 11.78 & - & 11.80 & - & 11.84 & - & 11.88 & - & 11.94 \\
\hline
\end{tabular}

$K=500, D=5200, h=10, p=20, h^{\prime}=5, i=0.1, \delta=0.0005$.

quality-adjusted stochastic lead-time models with and without investment in quality improvement are compared for uniformly distributed lead-time intervals of one through five weeks. In all cases the lot size based on the Quality Improvement Model are significantly smaller than those calculated based on the model without investment in quality improvement and significant cost savings on the order of 12 percent are realized. It is interesting to note that as lead-time variability increases optimal lot size and total cost increase. This suggests that a program aimed at simultaneously improving quality and reducing lead-time variability would have a synergistic effect. Similar results are obtained when lead-time follows a normal distribution that is truncated at $\pm 3 \sigma$, which may be seen in Table 5.3.

\section{Sensitivity analysis}

In this section we turn our attention to an investigation of the conditions under which investment in quality improvement is worthwhile. Specifically, we assume the probabilistic conditions in (2.8) and (2.9) are satisfied. Under this scenario investment is warranted if and only if $0 \leq \rho_{\text {imp }}<\rho_{0}$, which is the equivalent of requiring the optimal proportion of defective items, $\theta_{\text {imp }}$, to be nonnegative and strictly less than the original proportion of defective items, $\theta_{0}$. By substituting (4.8) for $\rho_{\text {imp }}$ in this relationship, we may solve for critical points for various parameters of interest in order to perform sensitivity analysis. These derived relationships can provide the manager with a yardstick to determine if investment in quality improvement would be worthwhile. 
Table 5.3. Optimal value for various normal lead-time variabilities.

\begin{tabular}{|c|c|c|c|c|c|c|c|c|c|c|}
\hline \multirow{3}{*}{ Variable } & \multicolumn{10}{|c|}{ Lead-time interval (weeks) } \\
\hline & \multicolumn{2}{|c|}{1} & \multicolumn{2}{|c|}{2} & \multicolumn{2}{|c|}{3} & \multicolumn{2}{|c|}{4} & \multicolumn{2}{|c|}{5} \\
\hline & QA & Imp & QA & Imp & QA & $\operatorname{Imp}$ & QA & $\operatorname{Imp}$ & QA & Imp \\
\hline$Q$ (units) & 942.22 & 894.37 & 944.48 & 896.52 & 948.23 & 900.08 & 953.46 & 905.04 & 960.14 & 911.39 \\
\hline$\theta$ & 0.20 & 0.0447 & 0.20 & 0.0446 & 0.20 & 0.0444 & 0.20 & 0.0442 & 0.20 & 0.0439 \\
\hline$\rho$ & 0.25 & 0.0468 & 0.25 & 0.0467 & 0.25 & 0.0465 & 0.25 & 0.0462 & 0.25 & 0.0459 \\
\hline $\mathrm{TC}(\$)$ & 6909.64 & 6095.95 & 6926.20 & 6110.07 & 6953.72 & 6133.52 & 6992.06 & 6166.20 & 7041.05 & 6207.96 \\
\hline $\begin{array}{l}\text { \%TC Sav- } \\
\text { ings over } \\
\text { SLT-QA }\end{array}$ & - & 11.776 & - & 11.783 & - & 11.795 & - & 11.811 & - & 11.832 \\
\hline
\end{tabular}

$K=500, D=5200, h=10, p=20, h^{\prime}=5, i=0.1, \delta=0.0005$.

Following the procedure outlined above, the critical point for demand is

$$
D>-K+\left[K^{2}+2(h+p) V+\left(\frac{h p i V}{\delta^{2} \rho_{0}^{2} h^{\prime 2}}\right)\right]^{1 / 2} .
$$

Thus, when demand is greater than the right-hand side of (6.1) it pays to invest. Similarly, the critical point for interest rate is

$$
i<\left[\frac{\delta^{2} \rho_{0}^{2} h^{\prime}(h+p)}{h p}\left(2 D K+(h+p) D^{2} V-2\right)\right]^{1 / 2} .
$$

Thus, when the interest rate is less than the right-hand side of (6.2) it pays to invest. Finally, the critical point for the lead-time variance is

$$
V>\frac{1}{(h+p) D^{2}}\left[\frac{h p i^{2}}{(h+p) \delta^{2} \rho_{0}^{2} h^{\prime 2}}+2(1-D K)\right] .
$$

Thus, when lead-time variance is greater than the right-hand side of (6.1) it pays to invest.

For each of these relationships we examine their sensitivity to a single parameter, holding all others constant. We examined a number of cases for which all parameter values are the same as in the sample problem. Critical points for demand show the quality improvement model to be quite robust. As the uniformly distributed lead-time interval increases from one to five weeks, the lower bound on demand for which the model remains optimal increases slightly from one unit to approximately four units. For the following situations we used a uniformly distributed lead-time over a four week interval. As the setup cost, $K$, increases from $\$ 250$ per setup to $\$ 1,500$ per setup, the lower bound on demand decreases from five units to about one unit. In terms of $\delta$, the percentage decrease in $\rho$ per dollar increase in investment, the lower bound on demand decreases from 622 units to less than one unit as $\delta$ increases from 0.000025 to 0.01 . This is logically consistent since in practice one would expect that as the marginal impact of investment on quality improvement increases, it would pay to engage in such an improvement program for even the smallest demand. For the holding cost for good items, $h$, the lower bound on demand increases from less than one unit to ten units as $h$ increases from $\$ 2$ to $\$ 40$. For the backorder cost, $p$, as it increases from $\$ 2$ to $\$ 40$, the lower bound on 
demand increases from less than one unit to five units. For the holding cost for defective units, $h^{\prime}$, as it changes from $\$ 2$ to $\$ 40$, the lower bound on demand decreases from 16 units to less than one unit. For all three inventory related unit costs the change in the lower bound for demand is essentially insignificant, indicating the robustness of the model. Further, in all three cases the change in the lower bound for demand is in the expected direction. That is, as the costs of holding a good item or backordering an item, $h$ and $p$, respectively, relative to that of holding a defective item, $h^{\prime}$, increase the lower bound on demand increases. This indicates that a stronger test for investment must be passed when the cost of good items is greater relative to the cost associated with defective items. While, on the other hand, as the cost of holding defective items increases relative to the costs associated with good items, the lower bound on demand decreases. This indicates that as the costs associated with defective items compose an increasing share of the burden, efforts at improving quality are worthwhile for even the smallest demand. However, in reality, for all intents and purposes, these points are moot due to the small changes in the lower bound that result. Finally, as $\theta$, the proportion defective, increases from 0.1 to 0.5 (resulting in $\rho$ increasing from 0.11 to 1.00), the lower bound on demand decreases from about 13 units to less than one unit. Again this is in the correct direction indicating that the poorer initial quality the greater the imperative to improve quality.

Now we turn our attention to the critical point for interest rate. As the uniformly distributed lead-time interval increases from one to five weeks, the upper bound on interest rate for which the model remains optimal increases slightly from 55.3 percent to 58.4 percent. As the setup cost, $K$, increases from $\$ 250$ per setup to $\$ 1,500$ per setup, the upper bound on interest rate increases from 41.9 percent to 96.8 percent. This makes intuitive sense since high setup costs mean large lot-sizes and a greater number of defectives per cycle. Thus one would be willing to pay higher investment costs to improve the quality and thus decrease the proportion of defectives. This indicates that a program of quality improvement should logically be accompanied by one aimed at reducing setup costs. The same argument can be made for the case of increasing demand. The results show that as demand increases from 1,250 units to 10,000 units the upper bound on the interest rate increases from 31.1 percent to 78.1 percent. An investigation of $\delta$ indicates the upper bound on interest rate increases from 2.9 percent to 114.6 percent as $\delta$ increases from 0.000025 to 0.01 indicating that the greater the impact of investment in quality improvement, the greater the upper bound of what one would be willing to pay to fund such a program. As for the inventory related costs, as $h$ increases from $\$ 2$ to $\$ 40$, the upper bound on interest rate decreases from 108.6 percent to 41.9 percent; as $p$ increases from $\$ 2$ to $\$ 40$ the upper bound on interest rate decreases from 112.1 percent to 53.5 percent; and as $h^{\prime}$ increases from $\$ 2$ to $\$ 40$ the upper bound on interest rate increases from 22.9 percent to 458.3 percent. Once again these results are in the expected direction. Finally, as $\theta$ increases from 0.1 to 0.5 the upper bound on interest rate increases from 25.5 percent to 229.1 percent. Again this is in the correct direction indicating that the poorer initial quality the greater the imperative to improve quality. These results indicate that the model is robust with respect to interest rate since all upper bounds are well above the prevailing cost of capital. 
A final interesting result is obtained from an investigation of the critical point for leadtime variance. Regardless of the values of the parameters previously discussed, investment in quality improvement is always warranted. That is, even for cases when lead-time is deterministic it is optimal to invest in quality improvement.

\section{Conclusion}

This paper presents an extension of the EOQ model with finite-range stochastic leadtimes in which the quality of items produced is considered. Specifically, a quality-adjusted model is developed in which the number of nondefective units in a lot is treated as a random variable. Relationships for economic lot-size, optimal total cost, optimal number of time units of demand satisfied by each order, and the optimal differential between placing an order and the start of time units that will be satisfied by the given order are derived. The work then proceeds to treat the proportion of defective items as a decision variable. A quality improvement model in which investment, according to a logarithmic investment function, to reduce the ratio of the proportion of defective to the proportion of nondefective items, is derived. Closed forms are developed for all the previously described decision variables in addition to the optimal quality ratio. Results of numerical examples indicate that significant savings can be realized by investing in quality improvement. The results of sensitivity analysis relating to demand, interest rate, and lead-time variability show the quality improvement model to be quite robust and representative of practice.

\section{References}

[1] T. C. E. Cheng, EPQ with process capability and quality assurance considerations, J. Oper. Res. Soc. 42 (1991), no. 8, 713-720.

[2] D. Gross and A. Soriano, The effect of reducing lead time on inventory level-simulation analysis, Management Sci. 16 (1969), B61-B76.

[3] R. W. Hall, Zero Inventories, Dow Jones-Irwin, Illinois, 1983.

[4] E. Heard and G. Plossl, Lead times revisited, Production and Inventory Management 23 (1984), no. 3, 32-47.

[5] M. J. Liberatore, The EOQ model under stochastic lead time, Oper. Res. 27 (1979), no. 2, 391396.

[6] K. Moinzadeh and H. L. Lee, A continuous-review inventory model with constant resupply time and defective items, Naval Res. Logist. 34 (1987), no. 4, 457-467.

[7] M. J. Paknejad, F. Nasri, and J. F. Affisco, Defective units in a continuous review $(s, Q)$ system, International Journal of Production Research 33 (1995), no. 10, 2767-2777.

[8] - Investment in quality improvement in continuous-review $(s, Q)$ systems, 5th International Conference of the Decision Sciences Institute, Decision Sciences Institute, Athens, 1999.

[9] E. L. Porteus, Investing in reduced setups in the EOQ model, Management Sci. 31 (1985), no. 8, 998-1010.

[10] - Investing in new parameter values in the discounted EOQ model, Naval Res. Logist. Quart. 33 (1986), 39-48.

[11] Optimal lot sizing, process quality improvement and setup cost reduction, Oper. Res. 34 (1986), no. 1, 137-144.

[12] M. J. Rosenblatt and H. L. Lee, Economic production cycles with imperfect production processes, IIE Trans. 18 (1986), no. 3, 48-55. 
[13] G. P. Sphicas, On the solution of an inventory model with variable lead times, Oper. Res. 30 (1982), 404-410.

[14] G. P. Sphicas and F. Nasri, An inventory model with finite-range stochastic lead times, Naval Res. Logist. Quart. 31 (1984), no. 4, 609-616.

[15] C. E. Vinson, The cost of ignoring lead time unreliability in inventory theory, Decision Sciences 3 (1972), no. 2, 87-105.

Javad Paknejad: Department of BCIS/QM, Frank G. Zarb School of Business, Hofstra University, Hempstead, NY 11549-1340, USA

E-mail address: m.j.paknejad@hofstra.edu

Farrokh Nasri: Department of BCIS/QM, Frank G. Zarb School of Business, Hofstra University, Hempstead, NY 11549-1340, USA

E-mail address: farrokh.nasri@hofstra.edu

John F. Affisco: Department of BCIS/QM, Frank G. Zarb School of Business, Hofstra University, Hempstead, NY 11549-1340, USA

E-mail address: john.f.affisco@hofstra.edu 


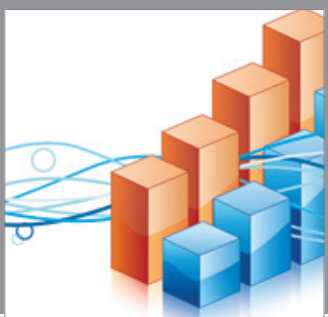

Advances in

Operations Research

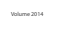

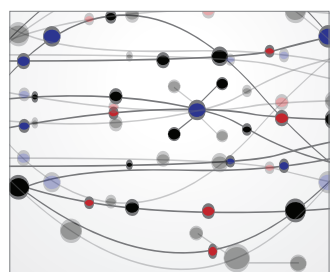

\section{The Scientific} World Journal
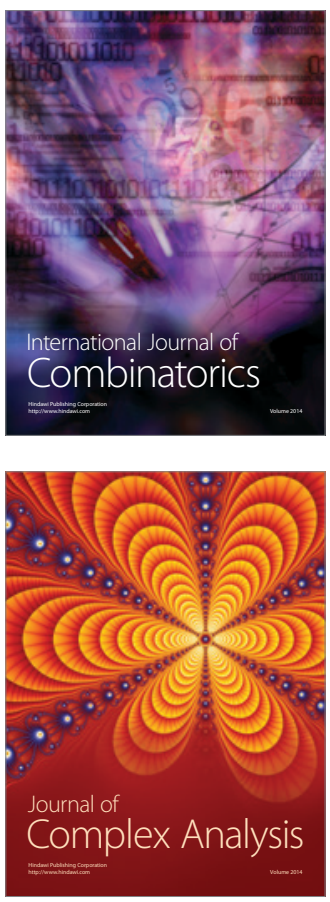

International Journal of

Mathematics and

Mathematical

Sciences
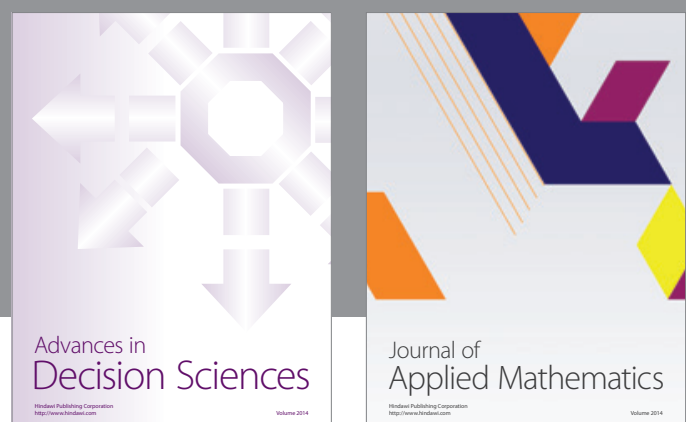

Journal of

Applied Mathematics
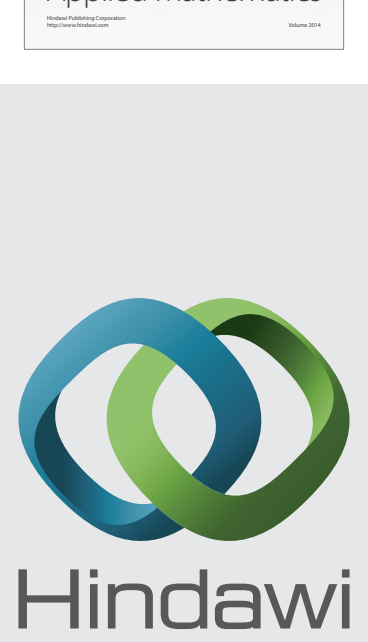

Submit your manuscripts at http://www.hindawi.com
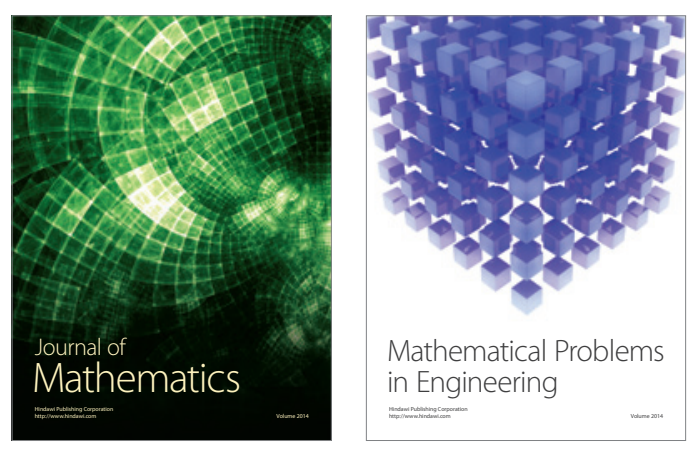

Mathematical Problems in Engineering
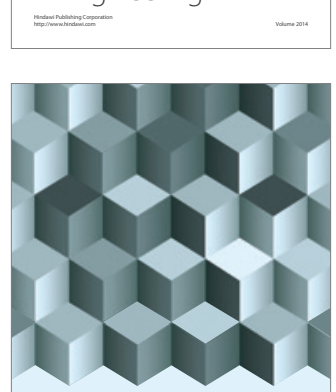

Journal of

Function Spaces
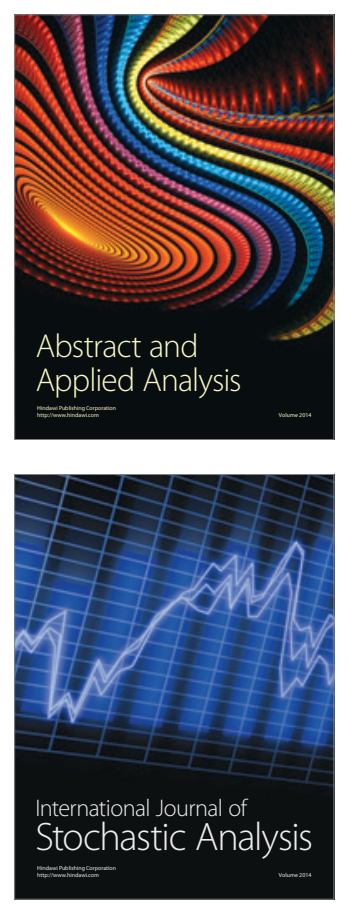

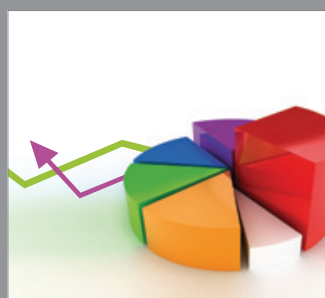

ournal of

Probability and Statistics

Promensencen
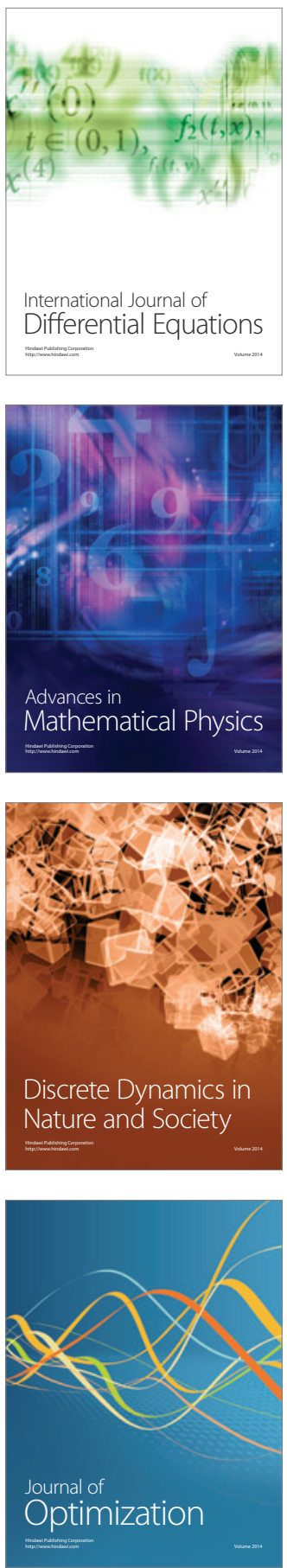